Journal of Advanced Research in Fluid Mechanics and Thermal Sciences

Journal homepage: www.akademiabaru.com/arfmts.html ISSN: 2289-7879

\title{
Effects of Heat and Mass Transfer on The Motion of Non- Newtonian Nanofluid Over an Infinite Permeable Flat Plate
}

\author{
Hameda M. Shawky ${ }^{1,}{ }^{*}$, Nabil T. M. Eldabe ${ }^{2}$, Kawther A. Kamel ${ }^{1}$, Esmat A. Abd-Aziz ${ }^{1}$ \\ Department of Mathematics, Faculty of Science, Al-Azhar University \\ Department of Mathematics, Faculty of Education, Ain Shams University
}

\section{ARTICLE INFO ABSTRACT}

\section{Article history:}

Received 5 November 2019

Received in revised form 22 December 2019

Accepted 22 December 2019

Available online 30 March 2020

Keywords:

Viscoelastic fluid; Nanofluid; Boundary layer motion; partial slip

\begin{abstract}
The motion of viscoelastic nanofluid flow with heat and mass transfer over a permeable flat plate under the action of uniform magnetic field is discussed. The effects of Brownian motion, thermophoresis and viscous and ohmic dissipations are considered. The system of equations describes the motion is converted to ordinary non-linear differential equations by using suitable transformations, and then solved numerically by using fourth order Runge-Kutta method with shooting technique. The obtained solutions are functions of the physical parameters of the problem. The effects of these parameters on the obtained solutions are discussed numerically and illustrated graphical to show that the parameters controlled the solutions.
\end{abstract}

Copyright @ 2020 PENERBIT AKADEMIA BARU - All rights reserved

\section{Introduction}

The flow over a shrinking sheet is a new field of research at present and few literatures is available on this area of research now. Wang [1] first studied a specific shrinking sheet problem. Much details Recently, Miklaveic and Wang [2] obtained the existence and uniqueness of the solution for steady viscous hydrodynamic flow over a shrinking sheet with mass suction. Hayat et al., [3] derive both exact and series solution describing the magnetohydrodynamic boundary layer flow of a second grad fluid over a shrinking sheet.

In the technical field Magnetohydrodynamic (MHD) flow in porous and non-porous media is of considerable interest due to its frequent occurrence in geothermal application and industrial technology, high temperature plasmas applicable to nuclear fusion energy conversion, liquid metal fluids and power generation systems. Chemical reaction can be classified as either homogeneous or heterogeneous processes. This depends on whether they occur at an interface or as a single-phase volume reaction.

\footnotetext{
* Corresponding author.

E-mail address: osama_96348@yahoo.com (Hameda M. Shawky)
} 
Heat and mass transfer together with chemical reaction play an important role in few representative fields of interest, as design of distribution of temperature and moisture over agricultural fields and groves of fruit trees, chemical processing equipment, formation and dispersion of fog, damage of crops due to freezing, food processing and cooling towers.

It is very important to study the heat source or sink in moving fluids in which deal with exothermic or endothermic chemical reaction and those concerned with dissociating fluids. For physical situations, the average behavior of heat absorption or generation can be expressed by some simple mathematical models because its exact modeling is quite difficult André Bakker [4] discussed the Heat Transfer Applied Computational Fluid Dynamics. Heat generation or absorption has been assumed to be constant, temperature-dependent or space dependent Nor Azwadi et al., [5] investigated the use of Fe3O4-H2O4 Nanofluid for Heat Transfer Enhancement in Rectangular Microchannel Heatsink. Sparrow and Cess [6] investigated the steady stagnation point flow and heat transfer in the presence of temperature dependent heat absorption. Later, Azim et al., [7] discussed the effect of viscous Joule heating on Magnetohydrodynamic (MHD) -conjugate heat transfer for a vertical flat plate in the presence of heat generation. One of the latest works is the study of the heat transfer characteristic in the mixed convection flow of a nanofluid along a vertical plate with heat source/sink studied by Rana and Bhargava [8].

It is now a well-accepted fact that many fluids of industrial and geophysical importance are nonNewtonian Abou-zeid et al., [9] investigated the flow of non-Newtonian power-law. Due to much attention in many industrial applications, such as the extrusion of plastic sheets, fabrication of adhesive tapes, glass-fiber production, metal spinning, and drawing of paper films, the research on the boundary layer behavior of a viscoelastic fluid over a continuously stretching surface keeps going where the velocity of a stretching surface is assumed to be linearly proportional to the distance from a fixed origin. McCormack and Crane [10] have provided comprehensive discussion on boundary layer flow caused by stretching of an elastic flat sheet moving in its own plane with a velocity varying linearly with distance. Several researchers namely, Gupta and Gupta [11], Dutta et al., [12] and Chen Char [13] extended the work of McCormack and Crane [10] by including the effects of heat and mass transfer under different situations. Later on, Rajagopal et al., [14] and Chang [15] presented an analysis on flow of viscoelasic fluid over a stretching sheet. The previous researchers used the case of no-slip condition. On the other hand, in certain circumstances, the partial slip between the fluid and the moving surface may occur in situations when the fluid is particulate such as emulsion, suspensions, foam and polymer solution. In these cases, the proper boundary condition is replaced by Navier's condition, where the amount of relative slip is proportional to local shear stress. Wang [16] discussed the partial slip effects on the planar stretching flow.

The transport of heat in a porous medium has considerable practical applications in geothermal system, crude oil extraction, and ground water pollution and also in a wide range of bio mechanical problems. The flow of a steady viscous fluid and heat transfer characteristics in a porous medium by considering different heating processes is studied by Vajravelu [17]. The problem for viscoelastic fluid flow and heat transfer in a porous medium over a stretching sheet was studied by Subhas and Veena [18]. The solution for both heat and mass transfer in hydromagnetic flow of a non-Newtonian fluid with heat source over an accelerated surface through porous medium has been found by Eldabe and Mohamed [19]. Eldabe et al., [20] investigated the thermal-diffusion and diffusion-thermo effects on mixed free-forced convection and mass transfer boundary layer flow of non-Newtonian fluid with temperature dependent viscosity.

Nor Azwadi et al., [21] studied numerical prediction of laminar nanofluid flow in rectangular microchannel. Akyildiz et al., [22] discussed nanoboundary layer fluid flows over stretching surface. Chamkha et al., [23] investigated the mixed convection flow of a nanofluid past a stretching surface 
in the presence of Brownain motion and thermophoresis effects. Das [24] studied Lie group analysis of stagnation-point flow of a nanofluid. Nanofluid flow over a shrinking sheet in the presence of surface slip was discussed by Das [25]. Recently heat transfer analysis of nanofluid over an exponentially stretching sheet was investigated by Nadeem et al., [26]. Thermal diffusion and diffusion thermo effects on the viscous fluid flow with heat and mass transfer through porous medium over a shrinking sheet was studied by Eldabe and Abu-Zeid [27]. Eldabe et al., [28] studied MHD boundary layer, flow with heat transfer of non-Newtonian Eyring-powel nanofluid past a stretching sheet. Hameda et al., [29] discussed the MHD flow with heat and mass transfer of nonNewtonian Williamson nanofluid over stretching sheet through porous medium. Williamson [30] The flow of pseudoplastic materials. Furthermore Eldabe et al., [31-32] studied micropolor Casson fluid motion and the non-Newtonian fluid flow over a semi-infinite moving vertical plate.

The main aim of this study is to investigate the boundary layer motion with heat and mass transfer of viscoelastic nanofluid over a permeable flat plate. Additionally, a similarity transforms is performed to reduce the governing equations to ordinary differential equations which are subsequently solved numerically using fourth order Runge-Kutta method with shooting technique. Results presented focus on how the magnetic field, partial slip, Brownian motion, thermophoresis and thermal radiation affect the heat and mass transfer characteristics of the flow.

\section{Mathematical Formulation}

Consider unsteady two-dimensional laminar flow of an incompressible electrically conducting viscoelastic nanofluid past a permeable shrinking sheet. The flow is subjected to a transverse magnetic field of strength $B_{0}$ which is assumed to be applied in the positive y-direction, normal to the surface. The velocity of the shrinking sheet is $u_{w}=-\frac{a x}{(1-b t)}$, where $\mathrm{a}, b>0$ are constants, $\mathrm{x}$ is the coordinate measured along the shrinking sheet and $t$ is time. The unsteady shrinking sheet has a uniform temperature and nanoparticle concentration $T_{w}$ and $C_{w}$, respectively, and these values are assumed to be greater than the ambient temperature $T_{\infty}$ and nanoparticale concentration $C_{\infty}$ respectively. The pressure gradient and external forces are neglected. The physical model and geometric coordinates are shown in Figure 1. Applying the Oberbeck-Boussinesq approximations [33] to the basic equations of an incompressible non-Newtonian nanofluid, we obtain

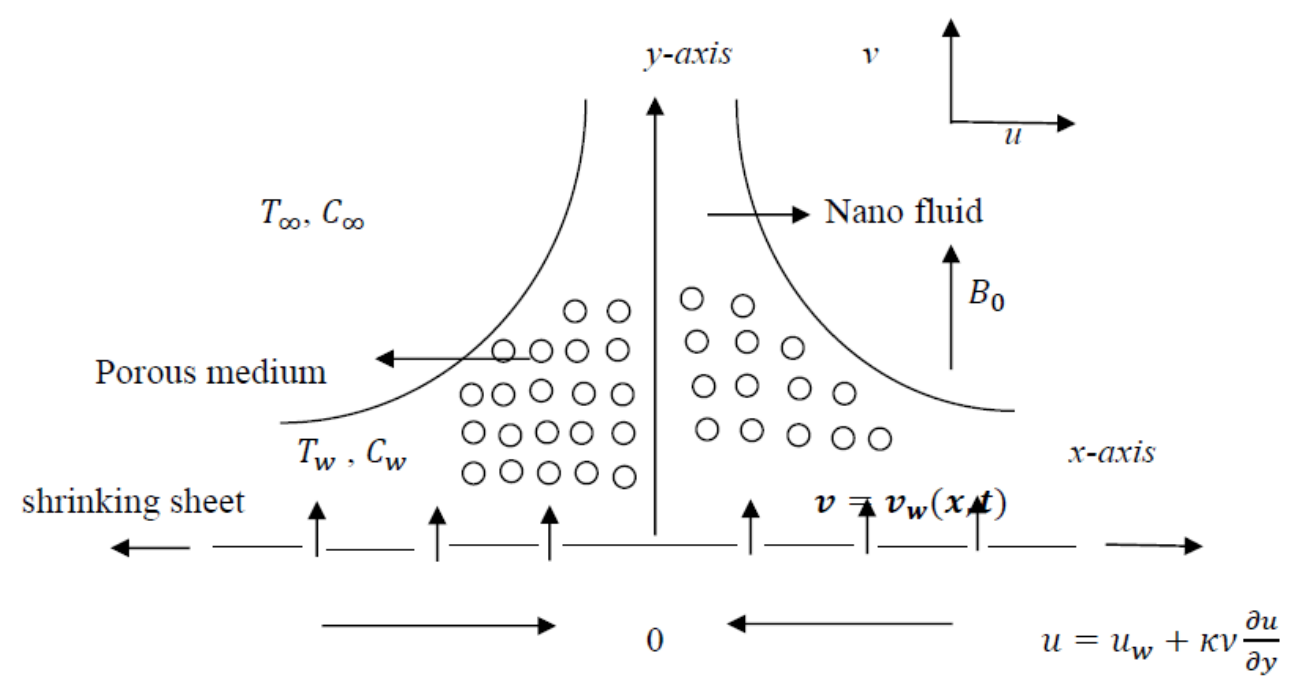

Fig. 1. Physical model and coordinates system 
$\frac{\partial u}{\partial x}+\frac{\partial v}{\partial y}=0$

$\frac{\partial u}{\partial t}+u \frac{\partial u}{\partial x}+v \frac{\partial u}{\partial y}=v \frac{\partial^{2} u}{\partial y^{2}}-\frac{k_{0}}{\rho_{f}}\left\{\frac{\partial^{3} u}{\partial y^{2} \partial t}+\frac{\partial}{\partial x}\left(u \frac{\partial^{2} u}{\partial y^{2}}\right)+\frac{\partial u}{\partial y} \frac{\partial^{2} v}{\partial y^{2}}+v \frac{\partial^{3} u}{\partial y^{3}}\right\}-\frac{\sigma}{\rho_{f}} B_{0}^{2} u-\frac{v u}{k^{\prime}}$,

$\frac{\partial T}{\partial t}+u \frac{\partial T}{\partial x}+v \frac{\partial T}{\partial y}=\alpha_{m} \frac{\partial^{2} T}{\partial y^{2}}+\tau\left[D_{B} \frac{\partial C}{\partial y} \cdot \frac{\partial T}{\partial y}+\frac{D_{T}}{T_{\infty}}\left(\frac{\partial T}{\partial y}\right)^{2}\right]+\frac{v}{c_{p}}\left(\frac{\partial u}{\partial y}\right)^{2}-\frac{k_{0}}{\rho_{f} c_{p}} \frac{\partial u}{\partial y}\left[\frac{\partial^{2} u}{\partial y \partial t}+\right.$

$\left.\frac{\partial}{\partial y}\left(u \frac{\partial u}{\partial x}+v \frac{\partial u}{\partial y}\right)\right]+\frac{\sigma B_{0}^{2}}{\rho_{f} c_{p}} u^{2}-\frac{1}{\rho_{f} c_{p}} \frac{\partial q_{r}}{\partial y}+\frac{Q_{0}}{\rho_{f} c_{p}}\left(T-T_{\infty}\right)$,

$\frac{\partial C}{\partial t}+u \frac{\partial C}{\partial x}+v \frac{\partial C}{\partial y}=D_{B} \frac{\partial^{2} C}{\partial y^{2}}+\frac{D_{T}}{T_{\infty}} \frac{\partial^{2} T}{\partial y^{2}}-k_{C}\left(C-C_{\infty}\right)$,

where $\mathrm{u}$ and $\mathrm{v}$ are the velocity components in the $\mathrm{x}$ and $\mathrm{y}$-direction respectively, $v$ is the kinematic viscosity, $\sigma$ is the electrical conductivity (assumed constant), $\rho_{f}$ is the density of the base fluid, $\alpha_{m}$ is the thermal diffusivity, $D_{B}$ is the Brownain diffusion coefficient, $D_{T}$ is the thermophoresis diffusion coefficient, $c_{p}$ is the specific heat capacity at constant pressure, $\tau$ is the ratio of the effective heat capacity of the ordinary fluid, $T$ is the fluid temperature and $C$ is the nanoparticle volume fraction.

The term $\frac{\sigma}{\rho_{f}} B_{0}^{2} u$ in the R.H.S. of Eq. (2) denotes the Lorentz force which arises from the interaction of the fluid velocity and the applied magnetic field. In writing Eq. (2), we have neglected the induced magnetic field since the magnetic Reynolds number for the flow is assumed to be very small. This assumption is justified for flow of electrically conductive fluids such as liquid metals e.g. mercury, liquid sodium, etc. (see Shercliff [34]). Eq. (3) depicts that heat can be transported in a nanofluid by convevtion, by conduction and also by virtue of nanoparticle diffusion, heat source/sink, viscous dissipation, Joule heating and radiation. The term $u \frac{\partial T}{\partial x}+v \frac{\partial T}{\partial y}$ is the heat convection, the term $\alpha_{m} \frac{\partial^{2} T}{\partial y^{2}}$ is the heat conduction, the term $\tau D_{B} \frac{\partial C}{\partial y} \cdot \frac{\partial T}{\partial y}$ is the thermal energy transport due to Brownian diffusion, the term $\tau \frac{D_{T}}{T_{\infty}}\left(\frac{\partial T}{\partial y}\right)^{2}$ is the energy transport due to thermophortic effect, the term $\frac{\sigma B_{0}^{2}}{\rho_{f} c_{p}} u^{2}$ is the energy transport due to Ohmic dissipation, $\frac{k_{0}}{\rho_{f} c_{p}} \frac{\partial u}{\partial y}\left[\frac{\partial^{2} u}{\partial y \partial t}+\frac{\partial}{\partial y}\left(u \frac{\partial u}{\partial x}+v \frac{\partial u}{\partial y}\right)\right]$ is the energy transport due to viscous dissipation effect, $\frac{Q_{0}}{\rho_{f} c_{p}}\left(T-T_{\infty}\right)$ is the heat source/sink and $\frac{1}{\rho_{f} c_{p}} \frac{\partial q_{r}}{\partial y}$ is the nanoparticle heat diffusion by radiation. Eq. (4) shows that the nanoparticles can move homogeneously within the fluid by the term $\left(u \frac{\partial C}{\partial x}+v \frac{\partial C}{\partial y}\right)$, but they also possess a slip velocity relative to the fluid due to Brownian diffusion $D_{B} \frac{\partial^{2} C}{\partial y^{2}}$, chemical reaction effect $k_{c}\left(C-C_{\infty}\right)$ and thermophoresis $\frac{D_{T}}{T_{\infty}} \frac{\partial^{2} T}{\partial y^{2}}$.

The boundary conditions for the velocity, temperature and concentration fields are given as follows:

$\left.\begin{array}{lr}u=u_{w}(x, t)+u_{\text {slip }}(x, t), \quad v=v_{w}(x, t), T=T_{w}, C=C_{w}, & \text { at } y=0, \\ u=0, \quad T=T_{\infty}, \quad C=C_{\infty}, \quad \frac{\partial u}{\partial y} \rightarrow 0, & \text { as } y \rightarrow \infty .\end{array}\right\}$

where 
$u_{w}=-\frac{a x}{(1-b t)}, \quad u_{\text {slip }}=L v \frac{\partial u}{\partial y}, \quad v_{w}=-\sqrt{\frac{a v}{(1-b t)}} S, \quad L=N(1-b t)^{\frac{1}{2}}$

Using Rosseland's approximation for radiation [35] we can write.

$\frac{\partial q_{r}}{\partial y}=-\frac{16 \sigma_{1} T_{\infty}^{3}}{3 K_{1}} \frac{\partial^{2} T}{\partial y^{2}}$

$v_{w}$ is the velocity of the mass transfer and $\mathrm{L}$ is the slip velocity factor, $\mathrm{s}$ is the constant wall mass transfer parameter with $\mathrm{S}>0$ for suction and $\mathrm{S}<0$ for injection, respectively. here $\mathrm{a}>0$ and $b \geq 0$ thus bt $<1$.

To obtain the similarity solutions of the Eqs. (1)-(4) with the boundary conditions in Eq. (5), we introduce the stream function $\psi$ defined in the usual way in terms of the velocity components $u=$ $\psi_{y}$ and $v=-\psi_{x}$, a similarity variable $\eta$ and the following similarity transformations are considered:

$\psi=\sqrt{\frac{a v}{1-b t}} x f(\eta), \quad \theta(\eta)=\frac{T-T_{\infty}}{T_{w}-T_{\infty}}, \quad \phi(\eta)=\frac{C-C_{\infty}}{C_{w}-C_{\infty}}, \quad \eta=y \sqrt{\frac{a}{v(1-b t)}}$

where $f(\eta), \theta(\eta)$ and $\phi(\eta)$ are the non-dimensional velocity, temperature and nanoparticles concentration, respectively. Using the transformation in Eq. (8), Eqs. (2)-(4), can be written in the following ordinary differential equations:

$f^{\prime \prime \prime}+f f^{\prime \prime}-f^{\prime 2}-A\left(f^{\prime}+\frac{1}{2} \eta f^{\prime \prime}\right)-\left(M+\frac{1}{K}\right) f^{\prime}+K_{0}\left[f^{\prime \prime 2}+f f^{(i v)}-2 f^{\prime} f^{\prime \prime \prime}-A\left(2 f^{\prime \prime \prime}-\frac{1}{2} \eta f^{(i v)}\right)\right]$

$=0$,

$\left(1-\frac{4}{3} R\right) \theta^{\prime \prime}+\left(f+A \frac{\eta}{2}\right) \operatorname{Pr} \theta^{\prime}+N b \operatorname{Pr} \phi^{\prime} \theta^{\prime}+N t \operatorname{Pr} \theta^{\prime 2}+\operatorname{Pr} E c f^{\prime \prime 2}+K_{0} \operatorname{Pr} E c\left[f^{\prime} f^{\prime \prime 2}+\right.$

$\left.\frac{A}{2}\left(3 f^{\prime \prime 2}+\eta f^{\prime \prime} f^{\prime \prime \prime}\right)-f f^{\prime \prime} f^{\prime \prime \prime}\right]+\operatorname{MPrEc} f^{\prime 2}+\operatorname{Pr} Q \theta=0$,

$\phi^{\prime \prime}+L e\left(f-\frac{A}{2} \eta\right) \phi^{\prime}+\frac{N t}{N b} \theta^{\prime \prime}-\operatorname{Pr} K_{C} \phi=0$,

subjected to the boundary conditions:

$\left.\begin{array}{l}f(0)=S, f^{\prime}(0)=-1+\delta f^{\prime \prime}(0), \quad \theta(0)=1, \quad \phi(0)=1, \quad \text { at } \eta=0 \\ f^{\prime}(\infty)=0, \quad f^{\prime \prime}(\infty)=0, \quad \theta(\infty)=0, \quad \phi(\infty)=0, \quad \text { as } \eta \rightarrow \infty .\end{array}\right\}$

where

$$
\begin{aligned}
& M=\frac{\sigma B_{0}^{2}(1-b t)}{a \rho_{f}}, \quad A=\frac{b}{a^{\prime}}, \quad R=\frac{4 \sigma_{1} T_{\infty}^{3}}{K_{1} \alpha_{m} \rho_{f} c_{p}}, \quad P r=\frac{v}{\alpha_{m}}, \quad v=\frac{\mu}{\rho_{f}}, \quad N b=\frac{\tau D_{B}\left(C_{w}-C_{\infty}\right)}{v}, \\
& N t=\frac{\tau D_{T}\left(T_{w}-T_{\infty}\right)}{v T_{\infty}}, \quad L e=\frac{v}{D_{B}}, \quad K=\frac{\rho_{f} k^{\prime} a}{\mu_{f}(1-b t)^{\prime}}, \quad K_{0}=\frac{k_{0} a}{\mu_{f}(1-b t)^{\prime}}, \quad K_{c}=\frac{k_{c}(1-b t)}{a}, Q=\frac{(1-b t) Q_{0}}{a}, \\
& E c=\frac{u_{w}^{2}}{c_{p}\left(T_{w}-T_{\infty}\right)}, \quad \delta=N \sqrt{a v} .
\end{aligned}
$$

The differentiation is with respect to $\eta$ and $A=\frac{b}{a}$ is the unsteadiness parameter, $\mathrm{M}$ is the dimensionless magnetic parameter, $\mathrm{R}$ is the thermal radiation parameter, $\mathrm{Pr}$ is the Prandtl number, 
$\mathrm{S}$ is the mass suction parameter, $\mathrm{Nb}$ is the Brownain motion parameter, $\mathrm{Nt}$ is the therophoresis parameter, $K_{c}$ is the chemical reaction parameter, Ec is the Eckert number, $\delta$ is the dimensionless velocity slip parameter, $K$ is the porous medium parameter, $K_{0}$ is the viscoelastic parameter, $\mathrm{Q}$ is the heat source or sink parameter and $L e$ is the Lewis number.

The skin-friction coefficient $C_{f}$, the local Nusselt number $N u_{x}$ and the Sherwoode number Sh are important physical parameters. Knowing the velocity field, the shearing stress at shrinking sheer can be obtained, in non-dimension form (skin-friction coefficient), as $C_{f}=\frac{\tau_{w}}{\rho_{f} U_{w}^{2}}$ where

$\tau_{w}=\left[\mu_{n f} \frac{\partial u}{\partial y}+k_{0}\left(\frac{\partial^{2} u}{\partial y \partial t}+\frac{\partial}{\partial y}\left(u \frac{\partial u}{\partial x}+v \frac{\partial u}{\partial y}\right)\right)\right]_{y=0}$,

or

$\left(R e_{x}\right)^{1 / 2} C_{f}=f^{\prime \prime}(0)-K_{0}\left(\frac{3}{2} A f^{\prime \prime}(0)+f^{\prime 2}(0) f^{\prime \prime}(0)+2 f^{\prime}(0) f^{\prime \prime}(0)-f(0) f^{\prime \prime \prime}(0)\right)$,

knowing the temperature field, the heat transfer coefficient at the sheet can be obtained, in dimensionless form, in terms of the Nusselt number, as:

$N u=\frac{x q_{w}}{k\left(T_{w}-T_{\infty}\right)}, q_{w}=-k\left(\frac{\partial T}{\partial y}\right)_{y=0}+\left(q_{r}\right)_{w}$,

or

$\left(R e_{x}\right)^{-1 / 2} N u_{x}=-\left(1+\frac{4}{3} R\right) \theta^{\prime}(0)$,

knowing the concentration field, the mass transfer coefficient at the sheet can be obtained, in nondimensional form, in terms of the Sherwood number, as:

$S h=\frac{x q_{m}}{D_{B}\left(C_{w}-C_{\infty}\right)^{\prime}} \quad q_{m}=-D_{B}\left(\frac{\partial C}{\partial y}\right)_{y=0}, \quad\left(R e_{x}\right)^{-1 / 2 S h_{x}}=-\phi^{\prime}(0)$,

where $R e_{x}=\frac{x U_{w}}{v_{f}}$ is the local Reynolds number based on shrinking velocity $u_{w}(x, t), q_{w}$ and $q_{m}$ are the wall heat and mass fluxes, respectively

\section{Results and Discussion}

The system of coupled, non-linear ordinary differential equations from Eqs. (9) to (11) subjected to the boundary condition in Eq. (12), are solved numerically using fourth order Runge-Kutta method with shooting technique [36-37]. The numerical computations have been carried out for different values of the parameters involved, namely, viscoelastic parameter $\mathrm{K}_{0}$, unsteadiness parameter $A$, magnetic field parameter $M$, mass suction parameter $S$, slip parameter $\delta$, Lewis number $L e$, heat source/sink parameter $Q$, the Brownian motion parameter $N b$, thermophoresis parameter $N t$, Prandtl number $P r$, the chemical reaction parameter $K_{c}$, the thermal radiation parameter $R$, the porous medium parameter $K$ and Eckert number parameter $E c$. The effects of these parameters on 
the velocity, temperature, the nanoparticles volume fraction, skin friction and the rate of heat and mass transfer are presented graphically in Figure 2-13.

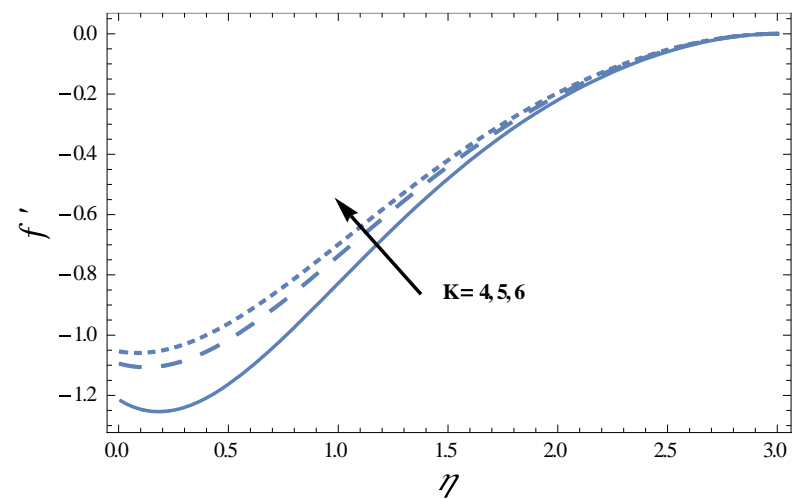

Fig. 2. The velocity distribution is plotted against $\eta$ for different values of $K$, with $\delta=0.01, K_{0}=1, \operatorname{Pr}=$ $0.5, N b=0.1, \mathrm{M}=0.3, N t=0.1, E c=0.3, \mathrm{Re}=$ $0.2, \mathrm{Q}=1, R=0.4, A=2.5, S=1, L e=1$ and $K c=$ 0.1

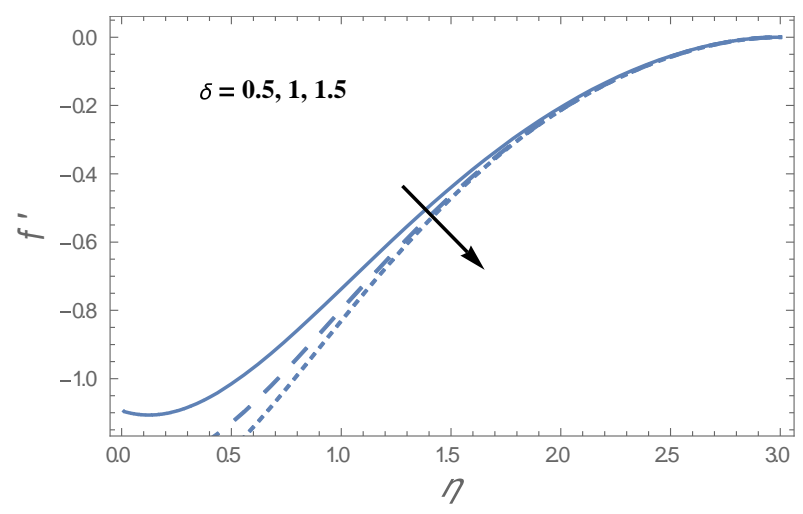

Fig. 4. The velocity distribution is plotted against $\eta$ for different values of $\delta$, with $A=2.5, K_{0}=1, \operatorname{Pr}=$ $0.5, N b=0.1, \mathrm{M}=0.5, N t=0.1, E c=0.3, \mathrm{Re}=$ $0.2, \mathrm{Q}=1, R=0.4, K=0.02, S=1, L e=1$ and $K c=$ 0.1

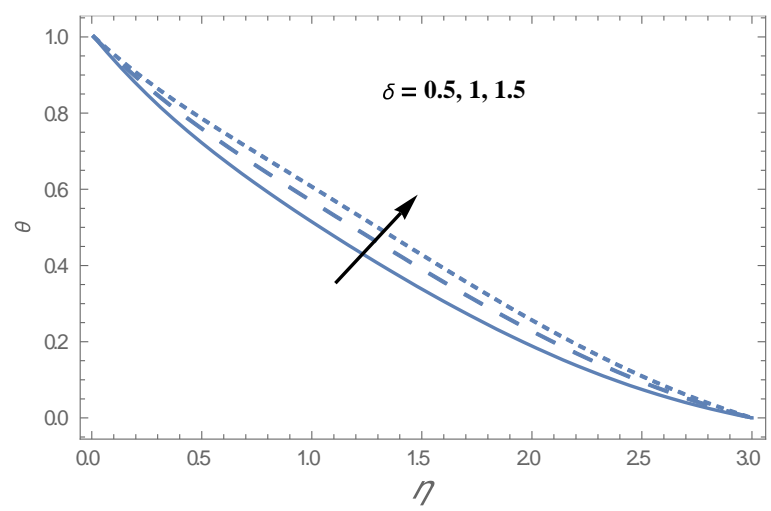

Fig. 6. The temperature distribution is plotted against $\eta$ for different values of $\delta$, with $A=2.5$, $K_{0}=1, \operatorname{Pr}=0.5, N b=0.1, \mathrm{M}=0.3, N t=0.1, E c=$ $0.3, \operatorname{Re}=0.2, \mathrm{Q}=1, R=0.4, K=10, S=1$, Le $=1$ and $K c=0.1$

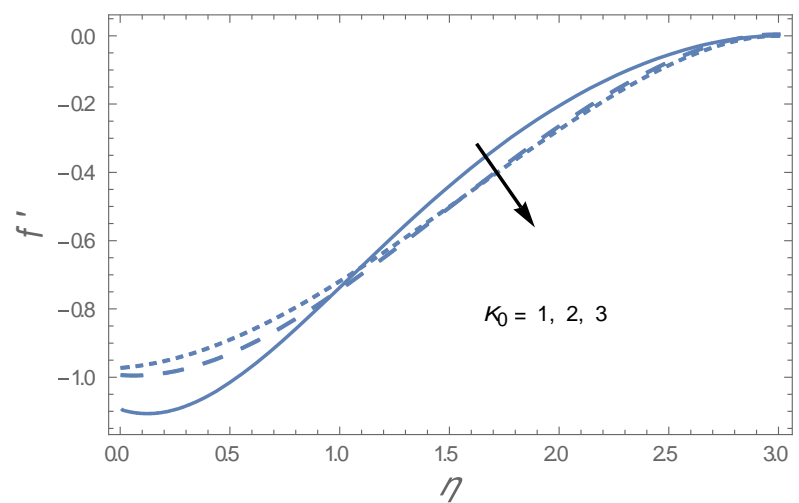

Fig. 3. The velocity distribution is plotted against $\eta$ for different values of $K_{0}$, with $\delta=0.01, A=2.5, \operatorname{Pr}=$ $0.5, N b=0.1, \mathrm{M}=0.3, N t=0.1, E c=0.3, \operatorname{Re}=0.2$, $\mathrm{Q}=1, R=0.4, K=10, S=1, L e=1$ and $K c=0.1$

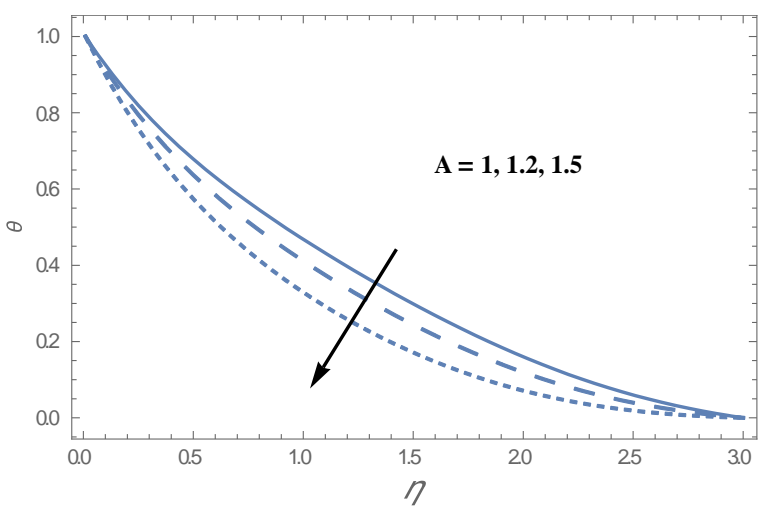

Fig. 5. The temperature distribution is plotted against $\eta$ for different values of $A$, with $\delta=0.01$, $K_{0}=1, \operatorname{Pr}=0.5, N b=0.1, \mathrm{M}=0.3, N t=0.1, E c=$ $0.3, \operatorname{Re}=0.2, \mathrm{Q}=1, R=0.4, K=10, S=1, L e=1$ and $K c=0.1$

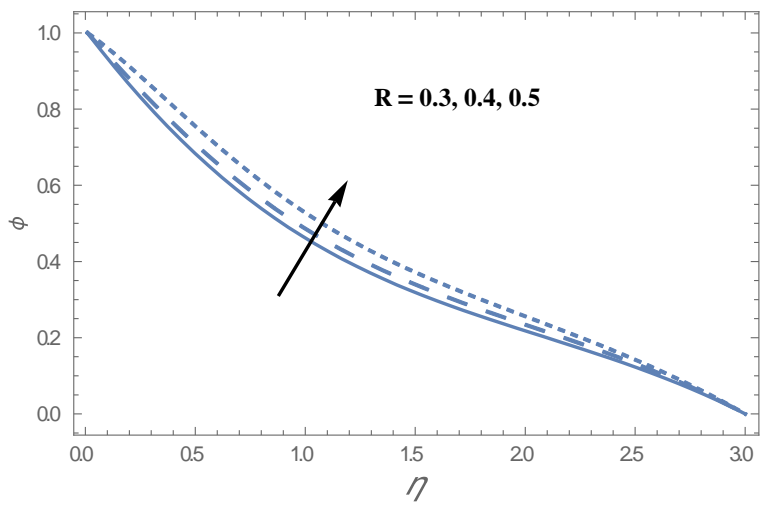

Fig. 7. The concentration distribution is plotted against $\eta$ for different values of $R$, with $\delta=0.01$, $K_{0}=1, \operatorname{Pr}=0.5, N b=0.1, \mathrm{M}=0.3, N t=0.1, E c=$ $0.3, \operatorname{Re}=0.2, \mathrm{Q}=1, A=2.5, K=10, S=1, L e=1$ and $K c=0.1$ 


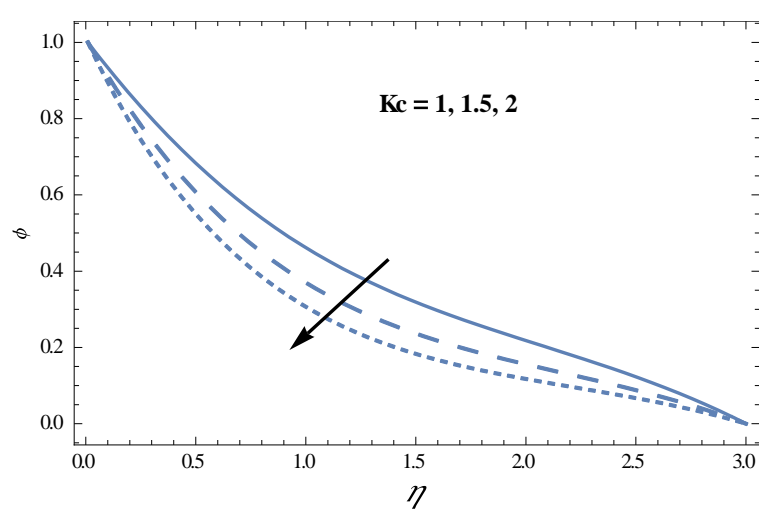

Fig. 8. The concentration distribution is plotted against $\eta$ for different values of $K c$, with $\delta=0.01$, $K_{0}=1, \operatorname{Pr}=0.5, N b=0.1, \mathrm{M}=0.3, N t=0.1, E c=$ $0.3, \operatorname{Re}=0.2, \mathrm{Q}=1, R=0.4, K=10, A=2.5$, Le $=1$ and $S=1$

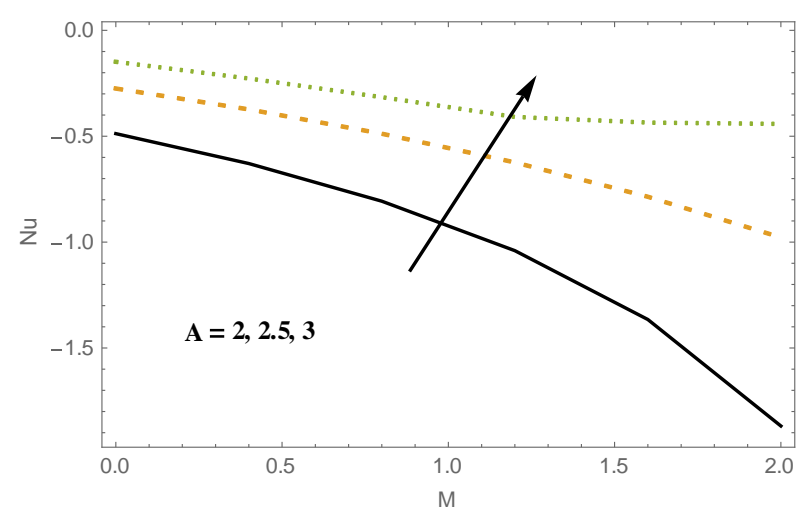

Fig. 10. The Nusselt number distribution is plotted against $\eta$ for different values of $A$, with $\delta=0.01, K_{0}$ $=1, \operatorname{Pr}=0.5, N b=0.1, \mathrm{M}=0.3, N t=0.1, E c=0.3$, $\operatorname{Re}=0.2, \mathrm{Q}=1, R=0.4, K=10, S=1, L e=1$ and $K c=0.1$

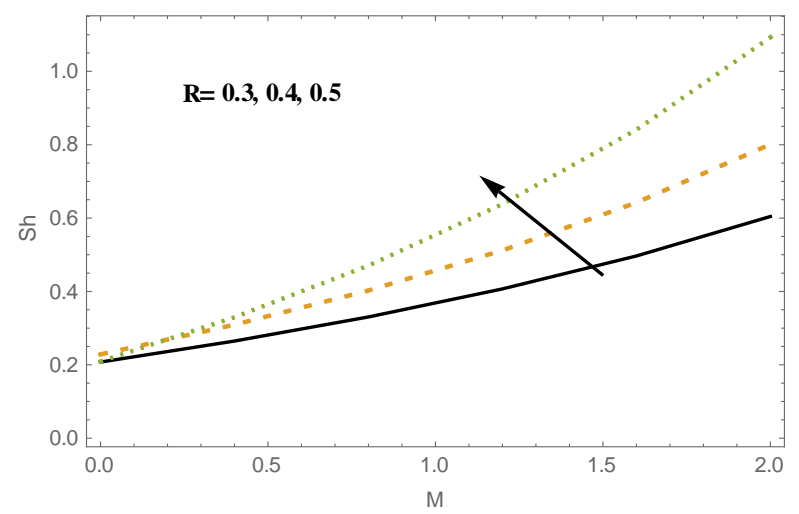

Fig. 12. The Sherwood number distribution is plotted against $\eta$ for different values of $R$, with $\delta=$ $0.01, K_{0}=1, \operatorname{Pr}=0.5, N b=0.1, \mathrm{M}=0.3, N t=$ $0.1, E c=0.3, \operatorname{Re}=0.2, \mathrm{Q}=1, A=2.5, K=10, S=1$, $L e=1$ and $K c=0.1$

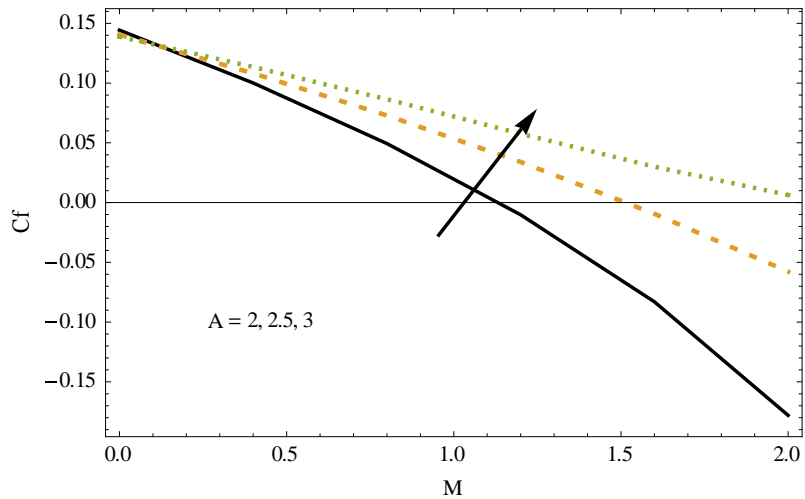

Fig. 9. The skin friction distribution is plotted against $\eta$ for different values of $A$, with $\delta=0.01, K_{0}=1, \operatorname{Pr}=$ $0.5, N b=0.1, \mathrm{M}=0.3, N t=0.1, E c=0.3, \operatorname{Re}=0.2$, $\mathrm{Q}=1, R=0.4, K=10, S=1, L e=1$ and $K c=0.1$

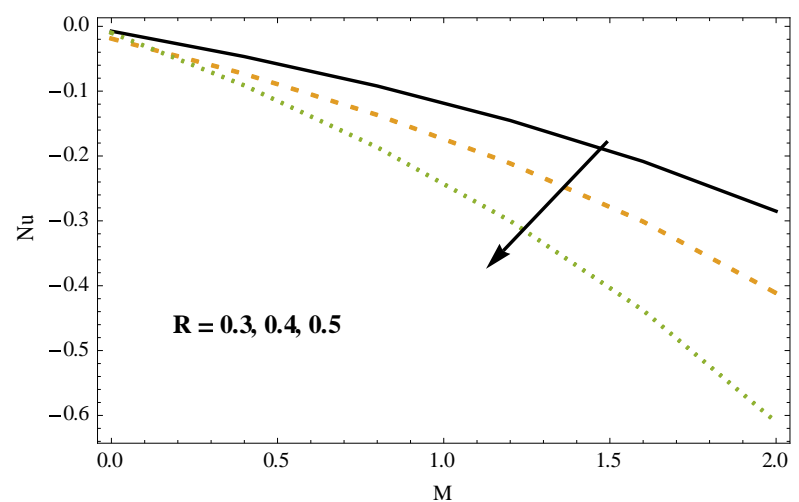

Fig. 11. The Nusselt number distribution is plotted against $\eta$ for different values of $R$, with $\delta=0.01, K_{0}$ $=1, \operatorname{Pr}=0.5, N b=0.1, \mathrm{M}=0.3, N t=0.1, E c=0.3$, $\operatorname{Re}=0.2, \mathrm{Q}=1, A=2.5, K=10, S=1, L e=1$ and $K c=$ 0.1

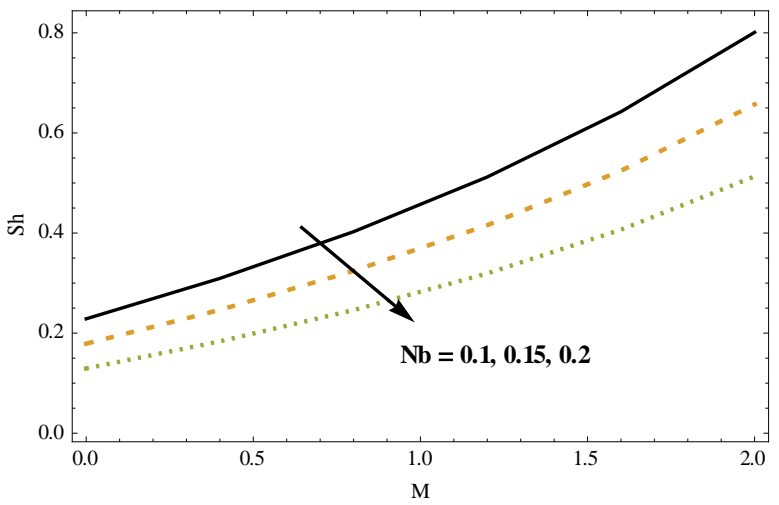

Fig. 13. The Sherwood number distribution is plotted against $\eta$ for different values of $N b$, with $\delta=0.01$, $K_{0}=1, \operatorname{Pr}=0.5, A=2.5, \mathrm{M}=0.3, N t=0.1, E c=$ $0.3, \operatorname{Re}=0.2, \mathrm{Q}=1, R=0.4, K=10, S=1, L e=1$ and $K c=0.1$ 
Figure 2 to 4 analyse the influences of $K, K_{0}$ and $\delta$ on $f^{\prime}(\eta)$. Figure 2 depicts that with the increase of porosity parameter $K$, the velocity increases. It is evident from Figure 3 that increasing values of viscoelastic parameter $K_{0}$ assists the motion of the liquid close to the shrinking sheet and opposes the motion of the liquid far away from the shrinking sheet. Figure 4 illustrates that as the slip parameter $\delta$ increases, the slip at the surface wall increases, as a result reaches to a smaller amount of penetration due to the shrinking surface into the fluid and the velocity component at the wall reduces with an increase in the slip parameter. It is clear also that as the magnitude of the unsteadiness parameter $A$ increases, $f^{\prime}(\eta)$ increases up to a point near the sheet but beyond this point opposite trend is observed. We observe that rising values of the magnetic field parameter $M$ increases the velocity profiles. This is due to the fact that the effect of horizontal magnetic field on electrically conducting fluid creates a drag force and develops the body force known as Lorentz force. This force helps to enhance the flow in shrinking case. The effect of the mass transfer parameter $S$ on the velocity profile $f^{\prime}(\eta)$, reveals that the velocity penetration into the fluid becomes shorter with the increase of $S$. Figures 5 and 6 are drawn to illustrate the effect of unsteadiness parameter $A$ and slip parameter $\delta$ on the temperature $\theta(\eta)$. Figure 5 reveals that the temperature at a point decreases as the magnitude of the unsteadiness parameter increases. This is due to the fact that the heat transfer rate increases with the increase in unsteadiness parameter which in turn reduces the temperature of the fluid. While Figure 6 indicates that an increase in slip parameter tends to increase the fluid temperature.

Also, the fluid temperature increases with increasing the porosity parameter $\mathrm{K}$, and also when the viscoelastic parameter $K_{0}$ is decreasing. Furthermore it is clear that the temperature decreases when both of magnetic parameter $M$, and thermal radiation $\mathrm{R}$ increases. This result can be explained by the fact that the decrease in the value of $R$ means a decrease in the Rosseland radiation absorptivity $k^{*}$ and due to this reason we have seen a fall in temperature profiles. Also, it is clear that the temperature profiles reduce with increasing Pr. It is clear when the Prandtl number Pr increases the thermal conductivity of the fluid reduces and consequently temperature of fluid decreases. The study of the effects of the Brownian motion parameter $\mathrm{Nb}$ and the therophoresis parameter $N t$ on fluid temperature clear that an increasing value of $\mathrm{Nb}$ and $\mathrm{Nt}$ enhances the temperature profiles. This is due to the fact that, different nanoparticles have different values of $N b$ and $N t$. This leads to different heat transfer rate. As a result, boundary layer thickness of the thermal field increases with enhancement in values of $\mathrm{Nb}$ and $\mathrm{Nt}$. Also, the temperature increases with Eckert number $E c$ due to the frictional heating, and it increases with the increasing of heat generation parameter $Q$ which increases the thermal state of the fluid. At last the increase of suction parameter $S$ is due to the decrease a temperature. The effect of suction parameter $S$ on $\theta(\eta)$ depicts that with the increase of suction parameter $S$, the temperature profiles decreases. The effects of the thermal radiation parameter $R$ and the chemical reaction parameter $K_{c}$ on the nanoparticles volume $\phi(\eta)$ fraction is illustrated through the Figures 7 and 8 . It is clear that $\phi(\eta)$ increases with $\mathrm{R}$ and decreases with $K_{c}$.

Also, other parameter effects on $\phi(\eta)$ are discussed. It is shown that, $\phi(\eta)$ increases with $A$, and with the Lewis number $L e$, due to the decreasing of mass diffusivity or the Brownian motion. Also, the nanoparticles volume fraction $\phi(\eta)$ increases with $S$ and decreases with $\delta$. Figure 9 , shows the relation between the skin friction coefficient $C_{f}$ and the unsteadiness parameter $A$, it is seen that $C_{f}$ increases with $A$. Also, the effects of $K_{0}$ and $K$ on $C_{f}$ are discussed. It is clear that $C_{f}$, enhanced for large values of $K_{0}$ and $K$. Figures 10 and 11 show the effects of $A$ and $R$ on Nusselt number $N u$. It is clear that $N u$ increases with $A$, while it decreases with $R$. Also, $N u$ decrease when both of $\operatorname{Pr}, N t, Q$ and $E C$ are increasing. Also, the effects of $R$ and $N b$ on the Sherwood number $S h$ are discussed. It is seen that $S h$ increases with $R$, and it decreases with $\mathrm{Nb}$. It is clear that $S h$ increases with both of $K$, $\mathrm{Nt}, E c$ and $L e$, while it decreases with both of $\operatorname{Pr}$ and $K_{c}$. 
Influence of porosity parameter $K$ and PrandtI number Pr on Sherwood number are explored that porosity parameter $K$ enhances the wall mass transfer while $\operatorname{Pr}$ reduce it. The influence of thermophoresis parameter $N t$ and Eckert number parameter Ec on Sherwood number. The behavior of it shows that the wall mass transfer increases for increasing thermophoresis parameter $N t$ and Eckert number parameter $E c$. The effect of chemical reaction parameter $K_{c}$ and Lewis number Le on Sherwood number are displayed that the Sherwood number enhances for increment in Lewis number Le but decreases for chemical reaction parameter $K_{c}$.

The most of the figures illustrated the effect of the physical parameters of the problem on the velocity, temperature concentration, skin friction, Nusselt and Sherwood numbers are excluded to save the space of the paper, and they are available under your request. This section discusses the results obtained from the surface pressure measurement study. The effects of angle of attack, Reynolds number and leading-edge bluntness are discussed in the next sub section.

\section{Conclusions}

A viscoelastic nanofluid flowing over an unsteady shrinking sheet in the presence of thermal radiation and heat generation are investigated numerically and the effect of the physical parameters of the problem illustrated graphically through a set of figures.

It is clear that the obtained solutions can be decrease or increase according to the variation values of the problem parameters.

The effect of the physical parameters on dimensionless velocity, temperature, nanoparticles volume fraction, skin friction, Nusselt and Sherwood numbers can be summarized as follows:

i. With the increase in the viscoelastic parameter $K_{0}$, unsteadiness parameter $A$, porosity parameter $K$, suction parameter $S$ and magnetic field parameter $M$, the velocity and nanoparticles concentration increase; however, the temperature of the fluid decreases.

ii. Temperature increases, but species concentration decreases when the strength of heat source parameter $Q$ and Brownian motion parameter increase.

iii. There is a decrease in the velocity and nanoparticles concentration, but temperature decrease with an increase in velocity slip parameter $\delta$.

iv. a rise in the radiation parameter $R$ enhances nanoparticles volume fraction as well as rate of mass transfer, but temperature of the fluid and rate of heat transfer decrease.

v. Both temperature and concentration fields are increased by increasing the values of a thermophoresis parameter $\mathrm{Nt}$.

vi. Temperature and rate of mass transfer are increasing functions of Eckert number $E c$.

vii. Concentration and mass transfer rate are found to increase with an increase in chemical reaction parameter $K_{c}$.

viii. Skin friction coefficient increases with an increase in unsteadiness parameter $A$, viscoelastic parameter $K_{0}$ and porosity parameter $K$.

ix. With the increase in unsteadiness parameter $A$ and porosity parameter $K$, the Nusselt number $\mathrm{Nu}$ and the Sherwood number $\mathrm{Sh}$ increases and decreases, respectively.

$\mathrm{x}$. By the increase of radiation parameter $R$, Prandtl number $P r$ and thermophoresis parameter $N$, the Nusselt number $\mathrm{Nu}$ and the Sherwood number $S h$ decreases and increases, respectively.

\section{References}

[1] Wang, C. Y. "Liquid film on an unsteady stretching surface." Quarterly of Applied Mathematics 48, no. 4 (1990): 601-610.

https://doi.org/10.1090/qam/1079908 
[2] Miklavčič, M., and C. Wang. "Viscous flow due to a shrinking sheet." Quarterly of Applied Mathematics 64, no. 2 (2006): 283-290.

https://doi.org/10.1090/S0033-569X-06-01002-5

[3] Hayat, T., Z. Abbas, and N. Ali. "MHD flow and mass transfer of a upper-convected Maxwell fluid past a porous shrinking sheet with chemical reaction species." Physics Letters A 372, no. 26 (2008): 4698-4704. https://doi.org/10.1016/i.physleta.2008.05.006

[4] Bakker, André. "Lecture 13-Heat Transfer (Applied Computational Fluid Dynamics)." Vorlesung. Fluent Inc (2006).

[5] Abubakar, S., CS Nor Azwadi, and A. Ahmad. "The use of Fe3O4-H2O4 nanofluid for heat transfer enhancement in rectangular microchannel heatsink." Journal Advanced Research Materials Science 23, no. 1 (2016): 15-24.

[6] Sparrow, Ephraim M., and R. D. Cess. "Temperature-dependent heat sources or sinks in a stagnation point flow." Applied Scientific Research 10, no. 1 (1961): 185.

https://doi.org/10.1007/BF00411912

[7] Azim, M. A., A. A. Mamun, and M. M. Rahman. "Viscous Joule heating MHD-conjugate heat transfer for a vertical flat plate in the presence of heat generation." International Communications in Heat and Mass Transfer 37, no. 6 (2010): 666-674. https://doi.org/10.1016/j.icheatmasstransfer.2010.02.002

[8] Rana, P., and R. Bhargava. "Numerical study of heat transfer enhancement in mixed convection flow along a vertical plate with heat source/sink utilizing nanofluids." Communications in Nonlinear Science and Numerical Simulation 16, no. 11 (2011): 4318-4334. https://doi.org/10.1016/i.cnsns.2011.03.014

[9] Abou-zeid, Mohamed Y., and Mona AA Mohamed. "Homotopy perturbation method for creeping flow of nonNewtonian power-law nanofluid in a nonuniform inclined channel with peristalsis." Zeitschrift für Naturforschung A 72, no. 10 (2017): 899-907. https://doi.org/10.1515/zna-2017-0154

[10] P. D. McCormack and L. J.Crane. "Fluid Dynamics." Academic Press, New York, NY, USA, (1973). https://doi.org/10.1016/B978-0-12-482250-4.50014-X

[11] Gupta, P. S., and A. S. Gupta. "Heat and mass transfer on a stretching sheet with suction or blowing." The Canadian Journal of Chemical Engineering 55, no. 6 (1977): 744-746. https://doi.org/10.1002/cjce.5450550619

[12] Dutta, B. K., P. Roy, and A. S. Gupta. "Temperature field in flow over a stretching sheet with uniform heat flux." International Communications in Heat and Mass Transfer 12, no. 1 (1985): 89-94. https://doi.org/10.1016/0735-1933(85)90010-7

[13] Char, Ming-I. "Heat transfer of a continuous, stretching surface with suction or blowing." Journal of Mathematical Analysis and Applications 135, no. 2 (1988): 568-580. https://doi.org/10.1016/0022-247X(88)90172-2

[14] Rajagopal, Kumbakonam R., T. Y. Na, and A. S. Gupta. "Flow of a viscoelastic fluid over a stretching sheet." Rheologica Acta 23, no. 2 (1984): 213-215. https://doi.org/10.1007/BF01332078

[15] Chang, Wen-Dong. "The nonuniqueness of the flow of a viscoelastic fluid over a stretching sheet." Quarterly of applied mathematics 47, no. 2 (1989): 365-366. https://doi.org/10.1090/qam/998108

[16] Wang, C. Y. "Flow due to a stretching boundary with partial slip-an exact solution of the Navier-Stokes equations." Chemical Engineering Science 57, no. 17 (2002): 3745-3747. https://doi.org/10.1016/S0009-2509(02)00267-1

[17] Vajravelu, K. "Flow and heat transfer in a saturated porous medium over a stretching surface." ZAMM-Journal of Applied Mathematics and Mechanics/Zeitschrift für Angewandte Mathematik und Mechanik 74, no. 12 (1994): 605614. https://doi.org/10.1002/zamm.19940741209

[18] Subhas, A., and P. Veena. "Visco-elastic fluid flow and heat transfer in a porous medium over a stretching sheet." International Journal of Non-Linear Mechanics 33, no. 3 (1998): 531-540. https://doi.org/10.1016/S0020-7462(97)00025-5

[19] Eldabe, Nabil TM, and Mona AA Mohamed. "Heat and mass transfer in hydromagnetic flow of the non-Newtonian fluid with heat source over an accelerating surface through a porous medium." Chaos, Solitons \& Fractals 13, no. 4 (2002): 907-917. https://doi.org/10.1016/S0960-0779(01)00066-2 
[20] Eldabe, Nabil T., A. G. El-Saka, and Ashraf Fouad. "Thermal-diffusion and diffusion-thermo effects on mixed freeforced convection and mass transfer boundary layer flow for non-Newtonian fluid with temperature dependent viscosity." Applied mathematics and computation 152, no. 3 (2004): 867-883.

https://doi.org/10.1016/S0096-3003(03)00603-9

[21] AbuBakar, Saidu Bello, Nor Azwadi Che Sidik, and Hong Wei Xian. "Numerical prediction of laminar nanofluid flow in rectangular microchannel." Journal of Advanced Research Design 50, no. 1 (2018): 1-17.

[22] Akyildiz, F. Talay, Hamid Bellout, Kuppalapalle Vajravelu, and Robert A. Van Gorder. "Existence results for third order nonlinear boundary value problems arising in nano boundary layer fluid flows over stretching surfaces." Nonlinear Analysis: Real World Applications 12, no. 6 (2011): 2919-2930.

https://doi.org/10.1016/i.nonrwa.2011.02.017

[23] Chamkha, Ali, Rama Subba Reddy Gorla, and Kaustubh Ghodeswar. "Non-similar solution for natural convective boundary layer flow over a sphere embedded in a porous medium saturated with a nanofluid." Transport in Porous Media 86, no. 1 (2011): 13-22.

https://doi.org/10.1007/s11242-010-9601-0

[24] Das, Kalidas. "Lie group analysis of stagnation-point flow of a nanofluid." Microfluidics and nanofluidics 15, no. 2 (2013): 267-274.

https://doi.org/10.1007/s10404-013-1147-3

[25] Das, Kalidas. "Nanofluid flow over a shrinking sheet with surface slip." Microfluidics and nanofluidics 16, no. 1-2 (2014): 391-401. https://doi.org/10.1007/s10404-013-1216-7

[26] Nadeem, Sohail, Rizwan UI Haq, and Zafar Hayat Khan. "Heat transfer analysis of water-based nanofluid over an exponentially stretching sheet." Alexandria Engineering Journal 53, no. 1 (2014): 219-224. https://doi.org/10.1016/i.aej.2013.11.003

[27] Eldabe, Nabil, and Mahmoud Abu Zeid. "Thermal diffusion and diffusion thermo effects on the viscous fluid flow with heat and mass transfer through porous medium over a shrinking sheet." Journal of Applied Mathematics 2013 (2013): 1-11. https://doi.org/10.1155/2013/584534

[28] Eldabe, Nabil TM, Ahmed Y. Ghaly, Mona AA Mohamed, and Mohamed SH Mahmoud. "MHD boundary layer chemical reacting flow with heat transfer of Eyring-Powell nanofluid past a stretching sheet." Microsystem Technologies 24, no. 12 (2018): 4945-4953.

https://doi.org/10.1007/s00542-018-3915-1

[29] Shawky, Hameda M., Nabil TM Eldabe, Kawther A. Kamel, and Esmat A. Abd-Aziz. "MHD flow with heat and mass transfer of Williamson nanofluid over stretching sheet through porous medium." Microsystem Technologies 25, no. 4 (2019): 1155-1169. https://doi.org/10.1007/s00542-018-4081-1

[30] Williamson, R. Vo. "The flow of pseudoplastic materials." Industrial \& Engineering Chemistry 21, no. 11 (1929): 1108-1111. https://doi.org/10.1021/ie50239a035

[31] Eldabe, N. T., G. M. Moatimid, A. A. Elshekhipy, and Naglaa F. Aballah. "Numerical simulation of the motion of a micropolar Casson fluid through a porous medium over a stretching surface." Thermal Science 00 (2019): 8-8. https://doi.org/10.2298/TSCI180604008E

[32] Eldabe, N. T., M. E. Gabr, A. A. Elshekhipy, and S. A. Zaher "The motion of non-Newtion nanofluid over a semiinfant moving vertical plat through porous medium with heat and mass transfer."

[33] Genieys, Stephane, and Marc Massot. "From Navier-Stokes equations to Oberbeck-Boussinesq approximation: a unified approach." (2001).

[34] Shercliff, John Arthur. "Textbook of magnetohydrodynamics." (1965).

[35] Hasimoto, Hidenori. "Boundary layer growth on a flat plate with suction or injection." Journal of the Physical Society of Japan 12, no. 1 (1957): 68-72. https://doi.org/10.1143/JPSJ.12.68

[36] Boldo, Sylvie, Florian Faissole, and Alexandre Chapoutot. "Round-off error and exceptional behavior analysis of explicit Runge-Kutta methods." IEEE Transactions on Computers (2019). https://doi.org/10.1109/TC.2019.2917902

[37] Summiya Parveen. "Numerical Solution of Non Linear Differential Equation by Using Shooting Techniques" International Journal of Mathematics And its Applications 4, no. 1-A (2016): 93-100. 